\section{Routine use of immunosuppressants is associated with mortality in hospitalised patients with COVID-19}

\author{
Phyo K. Myint*\# (D), Ben Carter*\#, Fenella Barlow-Pay, Roxanna Short, Alice G. Einarsson, \\ Eilidh Bruce (iD), Kathryn McCarthy, Alessia Verduri, Jemima Collins, James Hesford, \\ Frances Rickard, Emma Mitchell, Mark Holloway, Aine McGovern, Arturo Vilches-Moraga, \\ Philip Braude, Lyndsay Pearce, Michael Stechman, Angeline Price, Terence J. Quinn, \\ Enrico Clini, Susan Moug and Jonathan Hewitt, On behalf of COPE Study Team
}

\begin{abstract}
Background: Whilst there is literature on the impact of SARS viruses in the severely immunosuppressed, less is known about the link between routine immunosuppressant use and outcome in COVID-19. Consequently, guidelines on their use vary depending on specific patient populations.

Methods: The study population was drawn from the COPE Study (COVID-19 in Older People), a multicentre observational cohort study, across the UK and Italy. Data were collected between 27 February and 28 April 2020 by trained data-collectors and included all unselected consecutive admissions with COVID-19. Load (name/number of medications) and dosage of immunosuppressant were collected along with other covariate data.

Primary outcome was time-to-mortality from the date of admission (or) date of diagnosis, if diagnosis was five or more days after admission. Secondary outcomes were Day-14 mortality and time-to-discharge. Data were analysed with mixed-effects, Cox proportional hazards and logistic regression models using non-users of immunosuppressants as the reference group.

Results: In total 1184 patients were eligible for inclusion. The median (IQR) age was 74 (62-83), 676 (57\%) were male, and 299 (25.3\%) died in hospital (total person followup 15,540 days). Most patients exhibited at least one comorbidity, and $113(\sim 10 \%)$ were on immunosuppressants. Any immunosuppressant use was associated with increased mortality: aHR 1.87, 95\% Cl: 1.30, 2.69 (time to mortality) and aOR 1.71, 95\% Cl: 1.01-2.88 (14-day mortality). There also appeared to be a dose-response relationship.

Conclusion: Despite possible indication bias, until further evidence emerges we recommend adhering to public health measures, a low threshold to seek medical advice and close monitoring of symptoms in those who take immunosuppressants routinely regardless of their indication. However, it should be noted that the inability to control for the underlying condition requiring immunosuppressants is a major limitation, and hence caution should be exercised in interpretation of the results.
\end{abstract}

\section{Plain Language Summary}

Regular Use of Immune Suppressing Drugs is Associated with Increased Risk of Death in Hospitalised Patients with COVID-19

Background: We do not have much information on how the COVID-19 virus affects patients who use immunosuppressants, drugs which inhibit or reduce the activity of the immune
Ther Adv Drug Saf

2021, Vol. 12: 1-17

DOI: 10.1177/

2042098620985690

(c) The Author(s), 2021. Article reuse guidelines: sagepub.com/journalspermissions

Correspondence to: Jonathan Hewitt

Department of Geriatric

Medicine, 3rd Floor

Academic Centre,

Llandough Hospital,

Penlan Road, Penarth, CF64 2XX

Cardiff University, Cardiff, Wales, UK

HewittJ2Acardiff.ac.uk

Phyo K. Myint

Institute of Applied Health

Science, University of

Aberdeen, Aberdeen,

Scotland

Department of Medicine

for the Elderly, NHS

Grampian, Aberdeen,

Scotland

Ben Carter

Roxanna Short

Department of

Biostatistics \& Health

Informatics, King's College

London, England

Fenella Barlow-Pay

Royal Alexandra Hospital,

Paisley, Scotland

Alice G. Einarsson

Department of Medicine

for the Elderly, NHS

Grampian, Aberdeen,

Scotland

Eilidh Bruce

Institute of Applied Health

Science, University of

Aberdeen, Aberdeen.

Scotland

Kathryn McCarthy

James Hesford

Frances Rickard

Emma Mitchell

Mark Holloway

Philip Braude

North Bristol NHS Trust,

Bristol, England

Alessia Verduri

University of Modena and Reggio Emilia - Hospital

Policlinico, Modena,

Emilia-Romagna, Italy

Enrico Clini

University of Modena and

Reggio Emilia - Hospital

Policlinico, Modena,

Emilia-Romagna, Italy

Jemima Collins

Ysbyty Ystrad Fawr,

Aneurin Bevan University

Health Board, Wales,

Newport, UK 
Aine McGovern

Glasgow Royal Infirmary, Glasgow, Scotland

Arturo Vilches-Moraga Salford Royal NHS Trust and, Salford, UK

Lyndsay Pearce

Salford Royal NHS Trust and, Salford, UK

Manchester University, Manchester, England

Michael Stechman

University Hospital of

Wales, Cardiff, Wales, UK

Angeline Price

Manchester University,

Manchester, England

Terence J. Quinn

Cardiovascular and

Medical Sciences,

University of Glasgow,

Glasgow, Scotland

Susan Moug

University of Glasgow,

Glasgow, Scotland

*These authors (PKM \&

$\mathrm{BC}$ ) contributed equally

\#Joint first authors

system. There are various conflicting views on whether immune-suppressing drugs are beneficial or detrimental in patients with the disease.

Methods: This study collected data from 10 hospitals in the UK and one in Italy between February and April 2020 in order to identify any association between the regular use of immunosuppressant medicines and survival in patients who were admitted to hospital with COVID-19.

Results: 1184 patients were included in the study, and $10 \%$ of them were using immunosuppressants. Any immunosuppressant use was associated with increased risk of death, and the risk appeared to increase if the dose of the medicine was higher.

Conclusion: We therefore recommend that patients who take immunosuppressant medicines routinely should carefully adhere to social distancing measures, and seek medical attention early during the COVID-19 pandemic.

Keywords: Coronavirus, Covid-19, immunosuppressants, immunosuppression

Received: 15 September 2020; revised manuscript accepted: 12 December 2020

\section{Introduction}

SARS- CoV-2 (COVID-19) infection triggers local inflammatory and immune responses in the respiratory tract with resultant release of cytokines and priming of adaptive $\mathrm{T}$ and $\mathrm{B}$-cell immune responses. In most cases, this process helps to resolve the infection. However, a dysfunctional immune response can occur in some, causing systemic damage. ${ }^{1}$

Whilst it is well recognised that immunosuppression may render an individual more susceptible to viral illnesses, ${ }^{2}$ mild to moderate immunosuppression may have beneficial impact on outcomes of viral illnesses which can cause exaggerated immune response (cytokine storms) such as that caused by SARS-CoV-2 (commonly referred to as COVID-19). ${ }^{3}$

The beneficial effect of low-dose dexamethasone (glucocorticoids) on survival in severe COVID19 infection has been reported very recently. ${ }^{4}$ Prior to the current pandemic, studies have suggested that using inhaled corticosteroids may be a potentially therapeutic option in viral infections, especially coronaviruses in asthmatic patients, although good quality evidence is lacking. The potential benefit of immunosuppression in such illnesses may stem from their anti-inflammatory effects which could diminish the clinical expression of disease, including exaggerated immune response to viral illness.
Therefore, it is not surprising that immunosuppressants' usage during the COVID-19 pandemic is the centre of interest from several viewpoints with regard to: susceptibility to viral infection, ${ }^{1}$ plausibility of atypical presentation and un-recognised spread of infection, ${ }^{2}$ their role as a potential therapeutic option ${ }^{3}$, and their impact on prognosis in people who routinely require some form of immunosuppression for their pre-existing conditions. $^{5}$

The RECOVERY trial excluded patients where, in the opinion of the attending clinician, the patient would be at significant risk if he/she were to participate in the trial.

Evidence is lacking for those who use immunosuppressants for several conditions and have COVID-19 to a severity which requires hospital and/or intensive care admission. The primary aim of this study is to examine the association between immunosuppressant usage and in-hospital mortality, and length of hospital stay.

\section{Methods}

\section{Setting}

To enable timely collection of data, our existing network of clinical facilities with experience in collecting data for academic and service evaluation purposes Older Persons Surgical Outcome 
Collaborative (OPSOC; www.opsoc.eu) was utilised, with the addition of one Italian site. Data gathering occurred in 10 centres in the United Kingdom (Aberdeen Royal Infirmary, Glasgow Royal Infirmary, Nevill Hall Hospital, Abergavenny, Royal Alexandra Hospital, Paisley, Inverclyde Royal Infirmary, Inverclyde, Royal Gwent Hospital, Newport, Salford Royal Infirmary, Southmead Hospital, Bristol, University Hospital of Wales, Cardiff, and Ysbyty Ystrad Fawr, Caerphilly) and one Italian hospital (University Hospital of Modena Policlinico). Each of these hospitals delivered urgent, inpatient care to patients with COVID-19. Data were gathered between 27 February and 28 April 2020; however, UK data collection began on 6 March.

\section{Study design}

The COPE study (COVID-19 in Older People study) is a multicentre international observational study co-ordinated by OPSOC and provided this study population. The Health Research Authority (20/HRA/1898) granted permission to conduct the study in the United Kingdom, and in Italy this was granted by the Ethics Committee of Policlinico Hospital Modena (Reference 369/2020/OSS/AOUMO). Cardiff University sponsored the study.

Routinely recorded hospital data for patients with COVID-19 were collected prospectively using a standardised electronic case report format. This was supplemented by patient paper records, medication prescription charts and information from electronic records as required. All study personnel completed specific data collection training prior to capturing data. This was supervised locally by the site's principal investigator. We adhered to data protection policy in order to record data securely at each site, and each site subsequently transferred anonymised data to King's College London for statistical analysis.

\section{Participants}

Consecutive, unselected patients aged 18 years or older who were admitted to hospital with a diagnosis of COVID-19 were included. Diagnosis was made on the basis of laboratory-confirmed SARSCoV-2 positive swabs, or a clinical diagnosis (made by the clinical team at each site and based on signs, symptoms and/or radiology) consistent with COVID-19. No additional exclusion criteria were applied. In-patient admission lists were screened by clinical teams at each site for eligibility.

\section{Patient and public involvement}

No specific patient and public involvement was sought due to the nature of the research which required urgent data collection and analysis.

\section{Exposure}

Data on routine use of immunosuppressive agents including number and class of immunosuppressants that each patient was taking prior to admission were collected from admission records. Topical or inhaled immunosuppressants such as corticosteroid creams, enemas and inhalers were not included in these data. Immunosuppressants were grouped according to their therapeutic action as below:

- Glucocorticoids (e.g. prednisolone, dexamethasone)

- Antimetabolites (e.g. azathioprine, mycophenolate mofetil, leflunomide, methotrexate)

- T-cell inhibitors (e.g. ciclosporin, tacrolimus)

- Monoclonal antibodies (e.g. rituximab, infliximab)

- Cytotoxic agents (e.g. cyclophosphamide, chemotherapy regimens)

- Tyrosine kinase inhibitors (e.g. ibrutinib, afatinib)

Each patient's prescription was then defined as high or low dose for each drug they were taking (Supplementary Table 1). Patients' weights were not recorded in our data, hence where the literature suggested dose ranges in $\mathrm{mg} / \mathrm{kg}$ we assumed a $70 \mathrm{~kg}$ bodyweight. Data were collected for patients taking sulfasalazine, mesalazine and hydroxychloroquine; however, these are not widely felt to have significant immunosuppressive effects and thus these were coded separately. Supplementary Table 1 shows each immunosuppressant drug and their high/low-dose cut-off ranges based on prior studies throughout several specialities and current national guidelines which identify patient cohorts who are at higher risk of COVID-19 infection.

For the purposes of our analyses, we classified patients as having been prescribed any 
immunosuppressant versus none. In addition, we examined two different dosing variables. First, because it is known that patients on more than one immunosuppressant agent will be at a higher risk of infection due to cumulative effect, we also coded each patient's overall immunosuppressant load as none, low (one immunosuppressant) or high (two or more immunosuppressants). Second, we examined dose-response as follows: none; low dose (one immunosuppressant at a low or standard dose); moderate/high dose (one immunosuppressant at a high dose, or more than one immunosuppressant).

\section{Covariates}

We included variables with prognostic utility which included: patient age group (under 65, 6579 , or 80 years and over); sex; C-reactive protein (CRP) on admission; reduced renal function using estimated glomerular filtration rate (eGFR) on admission; smoking status (never, previous, or current), chronic obstructive pulmonary disease (COPD) on admission, frailty, and previous or current history of: coronary artery disease, diabetes mellitus, and hypertension. ${ }^{6-9}$ Frailty was measured using the pre-admission Clinical Frailty Score (CFS). ${ }^{10}$ The CFS is being used as an ordinal hierarchical scale that numerically ranks frailty from 1 to 9 , with a score of (1) being very fit, (2) well, (3) managing well, (4) vulnerable, (5) mildly frail, (6) moderately frail, (7) severely frail, (8) very severely frail and (9) terminally ill. The CFS has been used globally to aid clinical management $^{11}$ and frailty has been found to be independently associated with poorer outcomes in COVID-19 disease. ${ }^{12}$ Clinical Frailty Scores were grouped 1-2, 3-4, 5-6 and 7-9 for purposes of the analyses.

\section{End points}

The primary end point was the time to mortality from the date of admission (or date of diagnosis, if diagnosis was five or more days after admission). Secondary end points were Day-14 mortality and the time-to-discharge (herein described as the length of stay). For patients with a positive diagnosis of COVID-19 after 5 days from their date of the admission, their length of stay was calculated from the date of diagnosis so as not to be biased by length of stay independent of COVID status. All outcomes were assessed up to 28 April 2020.

\section{Statistical analysis}

Baseline demographic and clinical characteristics were partitioned by mortality, and patients who were prescribed an immunosuppressant versus those without. Time to mortality (primary end point) and length of stay (secondary endpoint) were analysed with mixed-effects multivariable Cox's proportional baseline hazards models. The analyses were fitted with a random intercept to account for hospital variation, and adjusted for the base model of: immunosuppressant prescribed (yes/no; and supplemented by the number of prescribed immunosuppressants); patient age group; sex; smoking status; CRP; diabetes; hypertension; coronary artery disease; reduced renal function $(e G F R<60)$; COPD; and the CFS. The adjusted hazard ratios (aHR) were estimated with associated $95 \%$ confidence intervals (95\% CI). The baseline proportionality assumption was tested visually with $\log -\log$ residuals. Each time-to-event analysis was reported with a Kaplan-Meier survival plot.

The secondary end point of Day-14 mortality was analysed using a mixed-effects multivariable logistic model, fitting each hospital as a random intercept effect, and adjusted with covariates consistent with the primary end point. The adjusted odds ratio (aOR) were estimated and presented with corresponding 95\% CI. Missing data were explored for patterns of missingness. Subgroup analyses were carried out to explore potentially moderating effects of immunosuppressant use within different subgroups stratified by: age group; sex; smoking status; diabetes; hypertension; coronary artery disease; and renal impairment. Analysis was carried out using Stata version $15^{13}$. Kaplan-Meier survival plots were visualised in $\mathrm{R},{ }^{14}$ with packages survival ${ }^{15}$ and survminer. ${ }^{16}$

\section{Sensitivity analysis}

A sensitivity analysis was carried out to assess the effect of only corticosteroids on the multivariable analyses for in-hospital mortality.

\section{Results}

A total of 1184 hospitalised adult patients with COVID-19 were included. Of them 1121 (94.7\%) were diagnosed via laboratory testing by PCR and the remaining 63 (5.3\%) via clinical diagnosis only. There were seven patients taking a single immunosuppressant of unknown dose, whose 
overall immunosuppression was imputed as 'low' for dose-response analysis. There were 222 cases of missing COPD that were imputed as not cases, 22 cases of missing smoking status, which were imputed as never smokers, and 32 with missing CRP, which were median imputed. There were no more than 14 patients missing for each of the remaining covariates. The complete case population was used within each analysis, and the number included shown as the population under investigation. The article and results have been published in Research Square pre-print server (DOI 10/21203/rs.3.rs-40131/v1).

\section{Descriptive data}

The median (IQR) age of the sample was 74 (6283 ) years, and 676 were male $(57 \%)$. The overall in-hospital mortality rate was $25.3 \%$ (299/1184), and this varied between $11.1 \%$ and $43.9 \%$ between sites (Table 1). The proportion of patients with pre-existing comorbidities were hypertension $(52.6 \%)$, diabetes $(26.3 \%)$, coronary artery disease $(23.1 \%)$, COPD $(11.2 \%)$, and $36.2 \%$ of them had reduced renal function at the time of admission. There were 113 patients who routinely used immunosuppressant constituting $9.5 \%$ of the sample $(11.2 \%$ in women and $8.3 \%$ in men) (Supplementary Table 2). Among the immunosuppressant users, corticosteroids were the most commonly prescribed immunosuppressant with 103 prescriptions ( $91.2 \%$ of users), followed by antimetabolites (37 prescriptions; $32.7 \%$ of users). With regards to steroids, 84 (74.3\% of immunosuppressant users) were prescribed a low dose, whilst $11(9.7 \%)$ were prescribed a high dose (unknown dose $n=8,7.1 \%$ ). A full breakdown of immunosuppressant prescription by type and dose is shown in Supplementary Table 3.

Prevalence of immunosuppressant use was more than two-fold among current smokers compared with never smokers ( $16.7 \%$ versus $8.1 \%)$. Of those patients that were not frail (CFS 1-4) $8.4 \%$ routinely used immunosuppressant compared with $10.7 \%$ in those who were frail. Full patient demographics and clinical characteristics are shown in Supplementary Table 2. In patients prescribed one or more immunosuppressants $31.9 \%$ and $40.9 \%$ died, compared with $24.4 \%$ of patients without any immunosuppressants during median (IQR) follow-up of 11 days (5-19) (total person follow-up 15,540 days).
In the crude analysis, it was found that use of any immunosuppressant agent was associated with increased mortality, hazard ratio $(95 \% \mathrm{CI})$ for time to mortality was $1.74,(1.23-2.46, p 0.002$; Table 2). There was also a likely load response: one drug versus none, HR 1.66, 95\% CI 1.13$2.46, p 0.01$; two or more drugs versus none, HR 2.03 95\% CI 1.03-3.98, p 0.04 (see Figure 1). Other important covariates which are known to be associated with an increased mortality in COVID-19 showed expected results. These included: older age (compared with under 65 year olds: patients aged 65-79, HR 3.28, $p<0.001$; patients aged over 80 years, HR 4.46, $p<0.001$ ); CRP (HR 1.003; $p<0.001$ ); reduced renal function (HR 2.08, $p<0.001$ ); coronary artery disease (HR 1.62, $p<0.001$ ), hypertension (HR $1.27, p$ 0.05), COPD (HR1.67, $p=0.002$ ), and frailty (CFS 3-4, HR 2.77, $p<0.001$; CFS $5-6$, HR 3.63, $p<0.001$; CFS 7-9, HR 5.83, $p<0.001$ ) (see Table 2).

In the multivariable analysis, we found that any immunosuppressant use was associated with an increased risk of mortality, aHR (95\% C) for time to mortality was $(1.87,1.30-2.69, p 0.001$; Table 2 ), with a likely load and dose-response: one immunosuppressant (versus none), aHR 1.77, $95 \%$ CI 1.18-2.65, $p=0.006$; two or more immunosuppressants (versus none), aHR $2.29,95 \%$ CI $1.15-4.53, p$ 0.02; low dose (versus none) aHR 1.88 , 95\% CI $1.23-2.88, p=0.004$; moderatehigh dose (versus none) aHR 1.85, 95\% CI 1.01$3.37, p=0.05$. Of the other covariates, it was also found that frailty (CFS $=3-4$, aHR $2.04,95 \% \mathrm{CI}$ $1.17-3.57, p$ 0.01; CFS 5-6, aHR 2.16, 95\% CI $1.21-3.88, p$ 0.009; CFS 7-9 aHR 3.22, 95\% CI 1.80-5.77, $p<0.001$ ), renal failure (aHR 1.40; $1.08-1.81, p 0.01$ ) and CRP (aHR 1.004, 95\% CI 1.003-1.005, $p<0.001$ ) also independently increased the risk of mortality.

For secondary endpoints, any immunosuppressant use was associated with a $70 \%$ increase in the odds of Day-14 mortality (aOR 1.71, 95\% CI 1.01-2.88, $p$ 0.04, Table 3), with a likely load response (one immunosuppressant, aOR 1.46, $95 \%$ CI $0.82-2.61, p 0.20$; two or more immunosuppressants aOR 3.34, 95\% CI 1.13-9.87, $p$ 0.03), dose-response (low dose, aOR 1.64, 95\% CI 0.90-3.00, $p=0.11$; moderate/high dose, aOR 1.90, 95\% CI 0.76-4.80, $p=0.17)$. Day-14 mortality was also associated with: older age (6575 versus under 65 ; aOR 2.91, $p<0.001$; over 80 
Table 1. Demographic and clinical characteristics, by primary end point of in-patient mortality.

\begin{tabular}{|c|c|c|c|}
\hline & Dead & Alive & Total \\
\hline Sites & $299(25.3)$ & $885(74.7)$ & 1184 \\
\hline Hospital A & $15(13.0)$ & $100(87.0)$ & 115 (9.7) \\
\hline Hospital B & $14(28.0)$ & $36(72.0)$ & $50(4.2)$ \\
\hline Hospital C & $34(22.2)$ & 119 (77.8) & 153 (12.9) \\
\hline Hospital D & $10(23.3)$ & 33 (76.7) & $43(3.6)$ \\
\hline Hospital E & 15 (12.2) & 108 (87.8) & $123(10.4)$ \\
\hline Hospital F & $23(14.9)$ & $131(85.1)$ & $154(13.0)$ \\
\hline Hospital G & $36(32.1)$ & 76 (67.9) & 112 (9.5) \\
\hline Hospital H & $108(43.9)$ & $138(56.1)$ & $246(20.8)$ \\
\hline Hospital I & $43(24.0)$ & $136(76.0)$ & $179(15.1)$ \\
\hline Hospital J & $1(11.1)$ & $8(88.9)$ & $9(0.8)$ \\
\hline \multicolumn{4}{|l|}{ Age } \\
\hline Under 65 years & $35(10.0)$ & $314(90.0)$ & 349 (29.5) \\
\hline 65-79years & 116 (27.9) & $300(72.1)$ & $416(35.1)$ \\
\hline Over 80 years & 148 (35.3) & $271(64.7)$ & 419 (35.4) \\
\hline \multicolumn{4}{|l|}{ Sex } \\
\hline Female & $119(23.4)$ & $389(76.6)$ & 508 (42.3) \\
\hline Male & $180(26.6)$ & 496 (73.4) & 676 (57.7) \\
\hline \multicolumn{4}{|l|}{ Smoking Status } \\
\hline Never smokers & 142 (22.5) & 488 (77.5) & $630(53.2)$ \\
\hline Ex-smokers & $127(29.1)$ & 309 (70.9) & $436(36.8)$ \\
\hline Current smokers & 22 (22.9) & $74(77.1)$ & $96(8.1)$ \\
\hline Missing & 8 & 14 & 22 \\
\hline CRP\&\& & $112,(58-181)$ & $68,(29-136)$ & $79,(33.5-150)$ \\
\hline \multicolumn{4}{|l|}{ eGFR $<60$} \\
\hline No & $135(18.1)$ & 612 (81.9) & $747(63.1)$ \\
\hline Yes & 159 (37.1) & 270 (62.9) & $429(36.2)$ \\
\hline Missing & 5 & 3 & 8 \\
\hline \multicolumn{4}{|l|}{ Hypertension } \\
\hline No & 122 (21.9) & 434 (78.1) & $556(47.0)$ \\
\hline Yes & 174 (27.9) & $449(72.1)$ & $623(52.6)$ \\
\hline
\end{tabular}


Table 1. (Continued)

\begin{tabular}{|c|c|c|c|}
\hline & Dead & Alive & Total \\
\hline Missing & 3 & 2 & 5 \\
\hline \multicolumn{4}{|c|}{ Coronary Artery Disease } \\
\hline No & $195(21.6)$ & 710 (78.5) & $905(76.4)$ \\
\hline Yes & $101(36.9)$ & $173(63.1)$ & $274(23.1)$ \\
\hline Missing & 3 & 2 & 5 \\
\hline \multicolumn{4}{|l|}{ COPD } \\
\hline No & $645(77.8)$ & $184(22.2)$ & $829(70.0)$ \\
\hline Yes & $89(66.6)$ & $44(33.1)$ & $133(11.2)$ \\
\hline Missing & 71 & 151 & 222 \\
\hline \multicolumn{4}{|l|}{ Diabetes } \\
\hline No & $203(23.4)$ & $665(76.6)$ & $868(73.3)$ \\
\hline Yes & $94(30.2)$ & $217(69.8)$ & $311(26.3)$ \\
\hline Missing & 2 & 3 & 5 \\
\hline \multicolumn{4}{|l|}{ Immunosuppressant } \\
\hline None & $261(24.4)$ & $810(75.6)$ & $1071(90.5)$ \\
\hline 1 & 29 (31.9) & $62(68.1)$ & $91(7.7)$ \\
\hline 2 & $9(40.9)$ & $13(59.1)$ & $22(1.9)$ \\
\hline \multicolumn{4}{|c|}{ Clinical Frailty Score (CFS) } \\
\hline 1 , Very fit & $4(4.8)$ & 79 (95.2) & $83(7.0)$ \\
\hline 2, Fit & 15 (10.3) & $131(89.7)$ & 146 (12.3) \\
\hline 3, Managing well & 29 (15.4) & $159(84.6)$ & $188(15.9)$ \\
\hline 4, Vulnerable & $40(27.8)$ & 104 (72.2) & $144(12.2)$ \\
\hline 5, Mildly frail & $31(21.7)$ & 112 (78.3) & $143(12.1)$ \\
\hline 6, Frail & 61 (32.3) & $128(67.7)$ & $189(16.0)$ \\
\hline 7, Severely frail & $70(36.1)$ & $124(63.9)$ & $194(16.4)$ \\
\hline 8, Very severely frail & 35 (51.5) & 33 (48.5) & $68(5.7)$ \\
\hline 9, Terminally ill & $11(45.8)$ & 13 (54.2) & $24(2.0)$ \\
\hline Missing & 3 & 2 & 5 \\
\hline
\end{tabular}




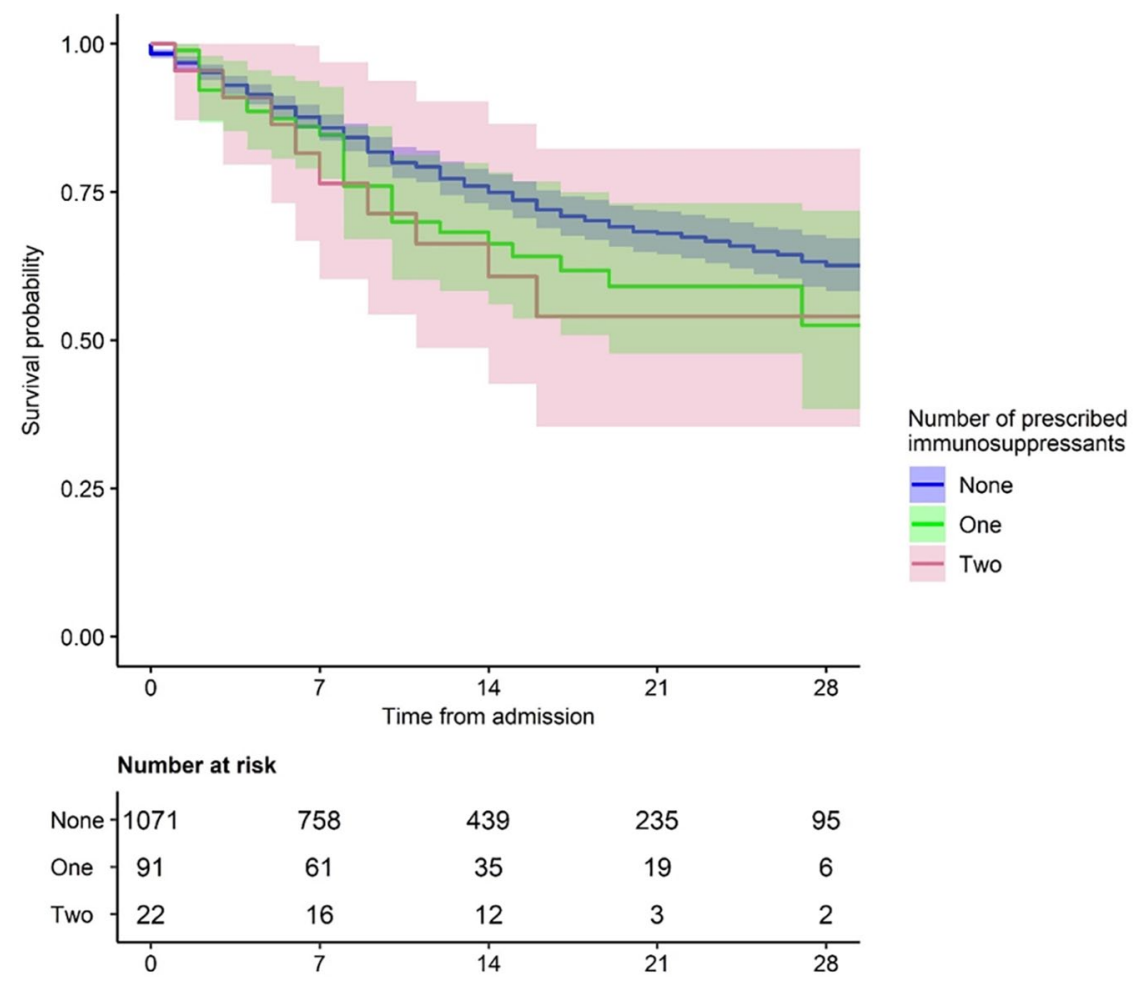

Figure 1. Kaplan-Meier Survival plot for patients prescribed with no, one or two immunosuppressants (immunosuppressant load), presented with 95\% confidence intervals.

Table 2. Primary end point: crude and adjusted time-to-mortality, from admission or diagnosis, for patients with a diagnosis five or more days after admission.

\begin{tabular}{|c|c|c|c|c|}
\hline & \multicolumn{2}{|c|}{ Crude hazard ratio (HR) } & \multicolumn{2}{|l|}{ Adjusted HR (aHR)\& } \\
\hline & \multicolumn{2}{|l|}{$(n=1150)^{\& \&}$} & \multicolumn{2}{|l|}{$(n=1136)^{\& \& \&}$} \\
\hline & $H R,(95 \%$ CI) & $p$-value & aHR, $(95 \% \mathrm{CI})$ & $p$-value \\
\hline \multicolumn{5}{|l|}{ Immunosuppressant } \\
\hline None (Ref) & \multicolumn{2}{|l|}{ Reference Category } & \multicolumn{2}{|l|}{ Reference Category } \\
\hline Any immunosuppressant & $1.74(1.23-2.46)$ & 0.002 & $1.87(1.30-2.69)$ & 0.001 \\
\hline One immunosuppressant ${ }^{\wedge}$ & $1.66(1.13-2.46)$ & 0.01 & $1.77(1.18-2.65)$ & 0.006 \\
\hline Two immunosuppressants^ & $2.03(1.03-3.98)$ & 0.04 & $2.29(1.15-4.53)$ & 0.02 \\
\hline Low dose^ & $1.87(1.19-2.75)$ & 0.003 & $1.88(1.23-2.88)$ & 0.004 \\
\hline Moderate/high dose^ & $1.61(0.91-2.84)$ & 0.33 & $1.85(1.01-3.37)$ & 0.05 \\
\hline \multicolumn{5}{|l|}{ Age } \\
\hline Under 65 & \multicolumn{2}{|l|}{ Reference Category } & \multicolumn{2}{|l|}{ Reference Category } \\
\hline $65-79$ & $3.28(2.20-4.90)$ & $<0.001$ & $2.23(1.43-3.49)$ & $p<0.001$ \\
\hline Over 80 & $4.46(2.99-6.65)$ & $<0.001$ & $3.11(1.95-4.97)$ & $p<0.001$ \\
\hline
\end{tabular}


Table 2. (Continued)

\begin{tabular}{|c|c|c|c|c|}
\hline & \multicolumn{2}{|c|}{ Crude hazard ratio (HR) } & \\
\hline & \multicolumn{2}{|l|}{$(n=1150)^{\& \&}$} & \multicolumn{2}{|l|}{$(n=1136)^{\& \& \&}$} \\
\hline & $\mathrm{HR},(95 \% \mathrm{CI})$ & $p$-value & aHR, $(95 \% \mathrm{CI})$ & $p$-value \\
\hline Sex (Female) & \multicolumn{2}{|l|}{ Reference Category } & \multicolumn{2}{|l|}{ Reference Category } \\
\hline Male & $1.01(0.79-1.28)$ & 0.96 & $1.14(0.88-1.48)$ & 0.32 \\
\hline Smoking status (Never) & \multicolumn{2}{|l|}{ Reference Category } & \multicolumn{2}{|l|}{ Reference Category } \\
\hline Ex-smokers & $1.30(1.01-1.66)$ & 0.04 & 1.02 (0.79-1.33) & 0.86 \\
\hline Current smokers & $1.01(0.64-1.60)$ & 0.97 & $1.04(0.64-1.70)$ & 0.88 \\
\hline $\mathrm{CRP}^{\$}$ & $1.003(1.002-1.004)$ & $<0.001$ & 1.004 (1.003-1.005) & $p<0.001$ \\
\hline Patients with diabetes & $1.22(0.95-1.58)$ & 0.12 & $1.10(0.83-1.44)$ & 0.52 \\
\hline $\begin{array}{l}\text { Patients with coronary artery } \\
\text { disease }\end{array}$ & $1.62(1.26-2.09)$ & $<0.001$ & 1.28 (0.98-1.69) & 0.08 \\
\hline Patients with hypertension & $1.27(1.00-1.61)$ & 0.05 & $1.01(0.78-1.30)$ & 0.97 \\
\hline Patients with COPD & $1.67(1.20-2.33)$ & 0.002 & $1.30(0.91-1.85)$ & 0.14 \\
\hline Patients with reduced renal function & $2.08(1.63-2.64)$ & $<0.001$ & $1.40(1.08-1.81)$ & 0.01 \\
\hline \multicolumn{5}{|l|}{ Clinical Frailty Scale } \\
\hline CFS 1-2 & \multicolumn{2}{|l|}{ Reference Category } & \multicolumn{2}{|l|}{ Reference Category } \\
\hline CFS 3-4 & $2.77(1.62-4.72)$ & $<0.001$ & $2.04(1.17-3.57)$ & 0.01 \\
\hline CFS 5-6 & $3.63(2.13-6.16)$ & $<0.001$ & $2.16(1.21-3.88)$ & 0.009 \\
\hline CFS 7-9 & $5.83(3.46-9.84)$ & $<0.001$ & $3.22(1.80-5.77)$ & $p<0.001$ \\
\hline \multicolumn{5}{|c|}{$\begin{array}{l}\text { COPD, chronic obstructive pulmonary disease; CRP, C-reactive protein } \\
\text { \&The multivariable mixed-effects Cox regression was adjusted for: age group; sex; smoking; CRP; diabetes; coronary } \\
\text { artery disease; eGFR, hypertension; COPD; and the Clinical Frailty Scale. } \\
\& \& 34 \text { Cases were not included in the analysis due to patient death on admission. } \\
\text { \&\&\&14 Cases were not included in the analysis due to missing covariate data-see Table } 1 . \\
\text { \$fitted as a slope parameter. } \\
\text { ^Fitted in replacement of "Any immunosuppressant" to demonstrate the load and dose-response, respectively. } \\
\text { Survival is estimated with a crude hazard ratio (HR), and adjusted Hazards Ratio (aHR), using a crude and adjusted mixed- } \\
\text { effects multivariable Cox proportional hazards regression. }\end{array}$} \\
\hline
\end{tabular}

versus under 65; aOR 4.66, $p<0.001)$; CRP (aOR 1.006, $p<0.001$ ); reduced renal function (aOR 1.66, $p$ 0.005); and increasing frailty (CFS 3-4 aOR 1.88, $p=0.08$; CFS 5-6 aOR 2.76, $p$ 0.006; CFS 7-9 aOR 5.74, $p<0.001)$. There was no association between any immunosuppressant use and the time to discharge (aHR 0.92, 95\% CI $0.68-1.26, p 0.62)$. Variables associated with an increased length of hospital stay were older age, higher CRP, and increasing level of frailty (see Table 3).

Supplementary Figures 1-3 show the forest plots demonstrating the adjusted hazards or odds ratios for different age groups, sex, smoking status and four major comorbidities. Overall, the results are largely consistent and as expected, and those variables which showed point estimates in an 
Table 3. Secondary end points. Outcome 1: day-14 mortality (left panel), and outcome 2: length of hospital stay (right panel).

\begin{tabular}{|c|c|c|c|c|}
\hline & \multicolumn{2}{|l|}{ Day-14 mortality } & \multicolumn{2}{|c|}{ Length of hospital stay } \\
\hline & \multicolumn{2}{|c|}{ Adjusted odds ratio (aOR) \& } & \multicolumn{2}{|c|}{ Adjusted hazard ratio (aHR) \& } \\
\hline & \multicolumn{2}{|l|}{$(n=1066)^{\& \& \&}$} & \multicolumn{2}{|l|}{$(n=1136)^{\& \&}$} \\
\hline & $\mathrm{HR},(95 \% \mathrm{CI})$ & $p$-value & aHR, $(95 \% \mathrm{CI})$ & $p$-value \\
\hline \multicolumn{5}{|l|}{ Immunosuppressant } \\
\hline None & \multicolumn{2}{|l|}{ Reference Category } & \multicolumn{2}{|l|}{ Reference Category } \\
\hline Any & $1.71(1.01-2.88)$ & 0.04 & $0.92(0.68-1.26)$ & 0.62 \\
\hline One immunosuppressant ${ }^{\wedge}$ & $1.46(0.82-2.61)$ & 0.20 & $1.03(0.74-1.44)$ & 0.84 \\
\hline $\begin{array}{l}\text { Two } \\
\text { immunosuppressants^ }\end{array}$ & $3.34(1.13-9.87)$ & 0.03 & $0.56(0.26-1.20)$ & 0.14 \\
\hline Low dose^ & $1.64(0.90-3.00)$ & 0.11 & $1.18(0.81-1.72)$ & 0.39 \\
\hline Moderate/high dose^ & $1.90(0.76-4.80)$ & 0.17 & $0.64(0.39-1.08)$ & 0.09 \\
\hline \multicolumn{5}{|l|}{ Age } \\
\hline Under 65 & Reference Category & & Reference Category & \\
\hline $65-79$ & $2.91(1.65-5.14)$ & $p<0.001$ & $0.72(0.57-0.91)$ & 0.005 \\
\hline Over 80 & $4.66(2.53-8.58)$ & $p<0.001$ & $0.49(0.37-0.66)$ & $p<0.001$ \\
\hline Sex (Female) & \multicolumn{2}{|l|}{ Reference Category } & \multicolumn{2}{|l|}{ Reference Category } \\
\hline Male & $1.17(0.82-1.66)$ & 0.39 & $1.00(0.83-1.21)$ & 0.99 \\
\hline Smoking status (Never) & \multicolumn{2}{|l|}{ Reference Category } & \multicolumn{2}{|l|}{ Reference Category } \\
\hline Ex-smokers & $0.93(0.65-1.35)$ & 0.72 & $0.93(0.77-1.13)$ & 0.49 \\
\hline Current smokers & 0.80 (0.41-1.59) & 0.53 & $0.96(0.67-1.37)$ & 0.83 \\
\hline CRP\$ & 1.006 (1.004-1.008) & $p<0.001$ & 0.997 (0.996-0.998) & $p<0.001$ \\
\hline Patients with diabetes & $1.11(0.76-1.62)$ & 0.60 & $0.99(0.80-1.23)$ & 0.92 \\
\hline $\begin{array}{l}\text { Patients with coronary } \\
\text { artery disease }\end{array}$ & $1.19(0.81-1.74)$ & 0.38 & $1.19(0.94-1.52)$ & 0.15 \\
\hline Patients with hypertension & $1.06(0.74-1.51)$ & 0.76 & $0.84(0.70-1.01)$ & 0.07 \\
\hline Patients with COPD & $1.50(0.90-2.48)$ & 0.12 & $0.99(0.73-1.35)$ & 0.96 \\
\hline $\begin{array}{l}\text { Patients with reduced renal } \\
\text { function }\end{array}$ & $1.66(1.17-2.36)$ & 0.005 & $0.88(0.71-1.08)$ & 0.22 \\
\hline
\end{tabular}

(Continued) 
Table 3. (Continued)

\begin{tabular}{|c|c|c|c|c|}
\hline & \multicolumn{2}{|l|}{ Day-14 mortality } & \multicolumn{2}{|c|}{ Length of hospital stay } \\
\hline & \multicolumn{2}{|c|}{ Adjusted odds ratio (aOR) \& } & \multicolumn{2}{|c|}{ Adjusted hazard ratio (aHR)\& } \\
\hline & \multicolumn{2}{|l|}{$(n=1066)^{\& \& \&}$} & \multicolumn{2}{|l|}{$(n=1136)^{\& \&}$} \\
\hline & $\mathrm{HR},(95 \% \mathrm{CI})$ & $p$-value & aHR, $(95 \% \mathrm{CI})$ & $p$-value \\
\hline \multicolumn{5}{|c|}{ Clinical Frailty Scale } \\
\hline CFS 1-2 & \multicolumn{2}{|c|}{ Reference Category } & \multicolumn{2}{|c|}{ Reference Category } \\
\hline CFS 3-4 & $1.88(0.94-3.78)$ & 0.08 & $1.06(0.83-1.35)$ & 0.64 \\
\hline CFS 5-6 & $2.76(1.33-5.71)$ & 0.006 & $0.71(0.53-0.95)$ & 0.02 \\
\hline CFS 7-9 & $5.74(2.75-11.98)$ & $p<0.001$ & $0.67(0.48-0.93)$ & 0.02 \\
\hline $\begin{array}{l}\text { COPD, chr } \\
\text { \&The multi } \\
\text { coronary a } \\
\text { \&\&\&104 cas } \\
\text { hospital. } \\
\text { \&\&34 Case } \\
\text { analysis du } \\
\text { NFitted in } r \\
\text { \$Fitted as } \\
\text { Day-14 mo } \\
\text { multivaria } \\
\text { admission } \\
\text { Hazards R }\end{array}$ & $\begin{array}{l}\text { ary disease; CRP, C- } \\
\text { gistic and cox regre } \\
\text { ion; COPD; and the } \\
\text { he analysis as the po } \\
\text { e analysis due to pat } \\
\text { ata-see Table } 1 . \\
\text { unosuppressant" to } \\
\text { ated with an adjuste } \\
\text { me } 2 \text { : Length of hos } \\
\text { s with a diagnosis fiv } \\
\text { with an adjusted mix }\end{array}$ & $\begin{array}{l}\text { protein } \\
\text { lere adjustec } \\
\text { =railty Scale. } \\
\text { s followed } \\
\text { th on admis } \\
\text { trate the loa } \\
\text { Ratio laOR) } \\
\text { (Right pane } \\
\text { re days after } \\
\text { ts multivari }\end{array}$ & $\begin{array}{l}\text { r: age group; sex; } \\
\text { for less than } 14 \text { Da) } 14 \text { cases we }\end{array}$ & $\begin{array}{l}\text { RP; diabetes; } \\
\text { and in } \\
\text { ded in the } \\
\text { ely. } \\
\text { mixed-effects } \\
\text { harge from } \\
\text { djusted } \\
\text { gression. }\end{array}$ \\
\hline
\end{tabular}

unexpected direction showed no statistically significant differences.

\section{Sensitivity analysis}

The sensitivity analysis, only including patients prescribed corticosteroids, reported a similar association between the medication and increased mortality. The aHR for in-hospital mortality was 1.96 (95\% CI 1.30- 2.95; $p=0.001)$ comparing no exposure with prescribed corticosteroids.

\section{Discussion}

This study has shown that prior, routine immunosuppressant use in unselected patients admitted to hospital with COVID-19 was associated with increased mortality risk, with a likely load and dose-response relationship. The observed effect sizes are large with a clinically meaningful increase in the risk of in-patient and day-14 mortality. Whilst the prevalence of immunosuppressant use is higher in women in this study, the mortality risk appears to be more pronounced in men, which is consistent with other COVID-19 studies. ${ }^{17} \mathrm{We}$ did not find any significant association between routine immunosuppressant usage and length of hospital stay. A further sensitivity analysis reports a similar association between corticosteroid use only and increased in-hospital mortality.

The prevalence of routine immunosuppressant use in our sample was approximately $10 \%$. The equivalent figures for general populations vary by countries, perhaps reflecting the different health care systems and challenges in obtaining accurate prescription data nationwide as well as frequency and chronicity of conditions that require use of immunosuppressants (e.g. autoimmune conditions) among the population. US studies estimated 2.7-6.2\% prevalent use of immunosuppressants among American adults. The former figure came from a study which estimated the prevalence through self-report (2013 data) from NHIS ${ }^{18}$ and the latter was derived from the national claims database MarketScan that included 47.2 million unique enrolees, representing 115 million person-years of observation during 2012-2017, and identified immunosuppressive conditions in 
$6.2 \%$ adults $18-64$ years of age. ${ }^{19}$ Therefore, apparently high prevalence usage of these drugs as routinely prescribed medication in our sample may be related to age and comorbidity profile of patients admitted to hospitals in UK and Italy.

Guidance on use of immunosuppressant agents during COVID-19 pandemic has been produced by a variety of national bodies and professional societies.

In transplant patients with confirmed or suspected COVID-19, the recommendation is to withhold immunosuppressant agents, with the exclusion of steroids. ${ }^{20}$ This recommendation stems from the balance between cyclophilins' potential antiviral activity via inhibition of peptidyl-prolyl isomerase (PPIase) activity ${ }^{21}$ versus their contribution to additional immunosuppression. Regarding the use of steroids in transplant patients, guidelines have been based on currently available evidence regarding corticosteroid treatment in COVID-19, which has suggested a possibility that steroid treatment may promote an exaggerated pro-inflammatory response or result in increased viral shedding; ${ }^{22}$ however following the recent pre-publication results from the RECOVERY trial ${ }^{4}$ demonstrating the prognostic benefit of short-term dexamethasone in severe COVID-19 infection, this guidance may evolve.

The National Institute for Health \& Care Excellence (NICE) in the UK recommends continuing corticosteroids, hydroxychloroquine, chloroquine, mepacrine, dapsone and sulphasalazine in those using these agents for dermatological conditions, but to consider temporarily stopping all other oral immunosuppressive therapies, novel small molecule immunosuppressants, biological therapies and monoclonal antibodies in the event of suspected or proven COVID-19 infection, ${ }^{23}$ advice which is reflected in guidelines from the American Association of Dermatologists. ${ }^{24}$

NICE recommends that patients with rheumatological, autoimmune, inflammatory and metabolic bone disorders are advised to continue with steroids, hydroxychloroquine and sulphasalazine but to temporarily stop other disease-modifying anti-rheumatic drugs, JAK inhibitors and biological therapies if they have COVID-19.25 The American College of
Rheumatology makes similar recommendations but includes sulphasalazine in the drugs that should be temporarily withheld. ${ }^{26}$ Hydroxychloroquine has been used to treat COVID-19 cases in hospitalised patients without rheumatological disorders; however, a recently reported observational study in the New England Fournal of Medicine failed to show significant benefit. $^{27}$ NICE has also produced COVID-19specific guidance on the delivery of systemic chemotherapeutic treatments, with the aim to 'deliver systemic anticancer treatment in different and less immunosuppressive regimens', including switching intravenous treatments to subcutaneous of oral alternatives, and decreasing the frequency of immunotherapy regimens. ${ }^{28}$ This is supported by evidence from a multicentre, retrospective study in cancer patients with COVID-19 infection where regression analysis revealed that receiving chemotherapy within 4 weeks before symptom onset was a significant risk factor for inhospital death. ${ }^{29}$

Current advice on the use of steroids, which were the most commonly used immunosuppressant in this study, is somewhat unclear. Many guidelines advice against abruptly stopping steroids (NICE 167, NICE 169, AAD); however, some guidelines recommend usual dose, ${ }^{30}$ or not to increase steroid dosing in mild symptomatic COVID-19 infection, whilst some recommend increasing dose or even giving high-dose steroids (methylprednisolone $1 \mathrm{mg} / \mathrm{kg}$ IV in severe cases). ${ }^{31}$ On the other hand, the British Society of Gastroenterology recommends avoidance of steroids if possible, or if not possible 'shielding' while prednisolone dose is $20 \mathrm{mg}$ or more. ${ }^{32}$ The cautious cessation of immunomodulators (azathioprine, mercaptopurine, thioguanine, methotrexate) in stable patients was advised with careful discussion on risk and benefit on a caseby-case basis, especially for those $>65$ or those with significant comorbidity. ${ }^{32}$ However, recent evidence suggests that the IL-17 blockers are not linked to increased mortality in association with COVID-19 disease, from two separate research groups in Europe and North America. ${ }^{33,34}$

The results of our sensitivity analysis with regard to corticosteroid immunosuppression alone are in keeping with the findings of other studies within the current literature base. The Global Rheumatology Alliance has found that steroid use 
$>10 \mathrm{mg}$ per day is associated with higher odds of hospitalisation, in comparison to other immunosuppressant drugs. ${ }^{35}$ Another study of patients with COVID-19 infection and underlying immune-mediated inflammatory disease found that the hospitalised patients were more likely to be prescribed corticosteroid or disease-modifying anti-rheumatic drugs. ${ }^{36}$

Given the current uncertainties around the use of these agents during the COVID-19 pandemic, our study provides novel insight and better understanding into the prognostic value of being a routine user of these agents in hospitalised patients with COVID-19. The findings are particularly important in busy clinical settings on several fronts. First, recognition by clinicians about the clinically important increased mortality risk associated with immunosuppressants should prompt them to carefully and closely monitor the patient's symptoms. Second, prognostic information is important for patients and their significant others, especially when hospitalised with COVID-19 infection, and even more so for patients who need immunosuppressant agents for their pre-existing medical condition(s) due to wide general knowledge of concern of impact of these drugs on outcome. Our results provide robust early (Day 14) and in-patient mortality risks associated with COVID-19 infection independently of other known important prognostic indicators including age, sex, frailty, smoking status, major comorbidities (hypertension, diabetes, renal function, and coronary artery disease) and disease severity marker (CRP). Our results also provide much needed evidence in the literature, which specifically lacks prognostic information generalisable to majority of the population, and therefore provide useful new knowledge to prescribers (general practitioners as well as specialists) and guideline developers, committees and panels.

Our results also provide evidence to support clinicians in advising the risk associated with routine use of these medications in their patients. The lessons learned may be particularly relevant and useful for future waves of the pandemic. It appears that in an unselected hospitalised patient population with COVID-19 infection, prior immunosuppression has important prognostic value. We are also first to report dose-response relationship between the number of agents and mortality. We did not observe significant differences in length of stay outcome, but this could be biased by early mortality.

Our findings should be considered in the light of a number of limitations. First, we did not collect data on the underlying condition for which immunosuppression was prescribed and were therefore unable to adjust for this, resulting in the potential for indicator bias. It is therefore possible that the underlying pathology is the true contributor to results including mortality. It therefore cannot be concluded from these results that immunosuppressants are the cause of high COVID-19 related mortality. Second, we did not check the compliance but it was likely to be high or very high during the incubation period and, aside from corticosteroids, these drugs would be unlikely to be started during a period of acute infection. Third, we did not collect detailed information on invasive management and dosing changes (withholding/increasing) of these agents, nor such immunosuppressant usage acutely for those who did not use immunosuppressant agents routinely as advised by some international ${ }^{37}$ and local guidelines. Nevertheless, current management strategies of COVID-19 in the UK and Europe are somewhat similar, and the internal relationship between the exposure and outcome we observed in this study is unlikely to be affected by this. Fourth, the study has intrinsic limitations associated with any observational study such as indication bias, that is, the impact of underlying condition requiring immunosuppressant therapy. However, the prospective relationship observed reduces the possibility of reverse causality, and selection bias was reduced by our unselected consecutive data collection methods.

Our study findings should be considered in the context of recently reported RECOVERY trial results which showed benefit of low-dose dexamethasone in seriously ill patients with COVID19.4 Some of the patients included in our study also participated in the RECOVERY trial in the UK. However, the allocation to trial arms would have happened randomly and thus this would not have major impact on our results. Eligibility criteria for RECOVERY states that if 'the attending clinician believes. . . that the patient should definitely be receiving one of the active drug treatment arms then that arm will not be available for randomisation for that patient', ${ }^{4}$ which would indicate that patients already prescribed 
corticosteroids would not be included in this arm of the trial. Thus, the results in our study may be an overestimation of the real impact associated with the routine use of immunosuppressants.

The study population was predominantly Caucasian; however, there is no reason to believe that the relationship between immunosuppressant use and outcome would be affected by ethnicity, and thus results are most likely to be generalisable regardless of ethnicity. Day-14 mortality was chosen over Day-7 mortality, as previous studies have demonstrated that the clinical course of COVID-19 disease comprises a longer time to mortality, particularly among patients who are ventilated. ${ }^{38,39}$

It is always possible that inaccuracies may have occurred in data collection; however, training in data collection was provided to all study personnel and the research team are experienced in collecting observation data in frail people from multiple UK sites (www.OPSOC.eu). These factors should have helped minimise bias. Even with a large sample, the proportion of people using immunosuppressants was relatively low (just over 100) and thus it was not possible to analyse the exposure-outcome relationships for each and individual classes of immunosuppressants. A final limitation is that patients were only included if admitted to hospital. That will have excluded patients who were discharged from or died in emergency departments, and excluded cases managed in the community. Nevertheless, the relationship we found between immunosuppressant usage and COVID-19 outcomes is unlikely to be different.

There are several strengths in our study. This was a large, multicentre prospective study involving front-line clinicians to gather a large dataset from patient records (paper form, electronic records or both) which minimised missing data. These data were collected from representative hospitals situated widely across England, Scotland and Wales, and $13 \%$ of the sample was derived from Italy. The demographic findings, such as the increased mortality demonstrated with a raised CRP and prevalence rates of comorbidities (hypertension, diabetes and ischaemic heart disease) are also in line with other estimates, suggesting that our data are comparable to other populations.
In summary, using a large unselected cohort of hospitalised adult patients with COVID-19 infection, we found that routine use of any immunosuppressant agents was associated with increased in-patient and early (within Day-14) mortality. The effect sizes observed are substantial, with almost doubling of poor outcomes. Although this study is limited by indicator bias, our study shows that this patient group is at increased risk of mortality regardless of whether the driver of worse outcomes is the immunosuppressant or the underlying condition for which it is prescribed. We therefore recommend that people who are on these agents for any condition abide by stringent social distancing measures, have a low threshold to seek early medical advice for COVID-19 symptoms, and for professionals to be aware of the prognostic impact of these agents and that close monitoring of worsening symptoms should be exercised in those who take immunosuppressants regardless of their indication.

\section{Acknowledgement}

We acknowledge the dedication, commitment, and sacrifice of the staff from participating centres across UK and Italy, two among the most severely affected countries in Europe. We gratefully acknowledge the contribution of our collaborators, National Institute of Health Research (NIHR), Health Research Authority (HRA) in the UK and Ethics Committee of Policlinico Hospital Modena, which provided rapid approval of COPE study and respective Institutions' Research and Development Offices and Caldicott Guardians for their assistance and guidance. We also thank COPE Study Sponsor, Cardiff University, Wales, UK.

\section{COPE Study Collaborators}

Professor Phyo Kyaw Myint - Professor of Medicine of Old Age

Dr Ben Carter - Senior Lecturer in Biostatistics

Dr Fenella Barlow-Pay - Specialist Registrar in Anaesthesia

Dr Roxanna Short - Research Associate

Dr Alice G Einarsson - Consultant Geriatrician

Dr Eilidh Bruce - Academic Foundation Trainee and Honorary Research Fellow

Miss Kathryn McCarthy - Consultant Surgeon \& Honorary Senior Lecturer 
Dr Alessia Verduri - Consultant Respiratory Physician

Dr Jemima Collins - Specialist Registrar in Geriatric Medicine

Dr Francis Rickard - Specialist Registrar Geriatric Medicine

Dr Emma Mitchell - Specialist Registrar Geriatric Medicine

Dr Mark Holloway - Intermediate Medical Trainee

Dr James Hesford - Clinical Fellow

Dr Aine McGovern - Consultant Geriatrician

Dr Arturo Vilches- Moraga - Consultant Geriatrician \& Honorary Senior Lecturer

Dr Philip Braude - Consultant Geriatrician

Miss Lyndsay Pearce - Consultant Surgeon

Mr Michael Stechman - Consultant Surgeon

Ms Angeline Price - Nurse Consultant

Dr Terence J Quinn - Clinical Senior Lecturer, Cardiovascular \& Medical Sciences

Professor Enrico Clini - Professor of Respiratory Medicine

Professor Susan J Moug - Consultant Surgeon

Dr Jonathan Hewitt - Clinical Senior Lecturer in Geriatric Medicine

Glasgow Royal Infirmary: Anna Fleck

Inverclyde Royal Infirmary: Carly Bisset, Ross Alexander

North Bristol Trust: Tarik Jichi, Sandeep Singh, Dolcie Paxton

Royal Alexandra Hospital: Norman Galbraith, Emma Bhatti, Jenny Edwards, Siobhan Duffy

Salford Royal Infirmary: Madeline Garcia, Shefali Sangani, Thomas Kneen, Thomas Lee

\section{University of Aberdeen: Mina Khezrian}

Ysbyty Yystad Fawr: Charlotte Davey, Sheila Jones, Kiah Lunstone, Alice Cavenagh, Charlotte Silver, Thomas Telford, Rebecca Simmons

\section{Authors Contribution}

$\mathrm{JH}$ and $\mathrm{KM}$ are PIs of COPE study. PKM conceived the study. AGE and FB-P performed literature search. BC and RS analysed the data.
PKM, BC, RS, AGE and FB-P drafted the paper. All authors contributed to data collection, interpretation of results and writing of the manuscript. $\mathrm{PKM}$ is the guarantor.

\section{Conflict of interest statement}

The authors declare that there is no conflict of interest.

\section{Funding}

The authors received no financial support for the research, authorship, and/or publication of this article.

\section{Study registration number}

IRAS project ID 281951

\section{Approval numbers}

UK Health Research Authority (Reference: 20/ HRA/1898)

Italy Ethics Committee of Policlinico Hospital Modena (Reference 369/2020/OSS/AOUMO)

\section{Protocol number}

SPON1815-20

ORCID iDs

Phyo K. Myint iD https://orcid.org/0000-00033852-6158

Eilidh Bruce (iD) https://orcid.org/0000-00015126-0943

\section{Supplemental material}

Supplemental material for this article is available online.

\section{References}

1. Tay MZ, Poh CM, Rénia L, et al. The trinity of COVID-19: immunity, inflammation and intervention. Nat Rev Immunol 2020; 20: 363-374.

2. Englund J, Feuchtinger T and Ljungman P. Viral infections in immunocompromised patients. Biol Blood Marrow Transplant 2011; 17(Suppl. 1): S2-S5.

3. Xu Z, Shi L, Wang $\mathrm{Y}$, et al. Pathological findings of COVID-19 associated with acute respiratory distress syndrome. Lancet Respir Med 2020; 8: $420-422$.

4. University of Oxford. RECOVERY. 2020 Nuffield department of population health, 
https://www.recoverytrial.net/files/recovery_ dexamethasone_statement_160620_v2final.pdf (accessed 22 June 2020).

5. Halpin DMG, Singh D and Hadfield RM. Inhaled corticosteroids and COVID-19: a systematic review and clinical perspective. Eur Respir f 2020; 55: 2001009.

6. Ruan Q, Yang K, Wang W, et al. Clinical predictors of mortality due to COVID-19 based on an analysis of data of 150 patients from Wuhan, China. Intensive Care Med 2020; 46: 846-848.

7. Guan W-J, Ni Z-Y, Hu Y, et al. Clinical characteristics of coronavirus disease 2019 in China. N Engl f Med 2020; 382: 1708-1720.

8. Zhou F, Yu T, Du R, et al. Clinical course and risk factors for mortality of adult inpatients with COVID-19 in Wuhan, China: a retrospective cohort study. Lancet 2020; 395 : 1054-1062.

9. Huang C, Wang Y, Li X, et al. Clinical features of patients infected with 2019 novel coronavirus in Wuhan, China. Lancet 2020; 395: 497-506.

10. Rockwood K, Song X, MacKnight C, et al. A global clinical measure of fitness and frailty in elderly people. CMAF 2005; 173: 489-495.

11. Hewitt J, Carter B, McCarthy K, et al. Frailty predicts mortality in all emergency surgical admissions regardless of age. An observational study. Age Ageing 2019; 48: 388-394.

12. Hewitt J, Carter B, Vilches-Moraga A, et al.; COPE Study Collaborators. The effect of frailty on survival in patients with COVID19 (COPE): a multicentre, European, observational cohort study. Lancet Public Health 2020; 5: e444-e451.

13. StataCorp. Stata statistical software: release 15 . College Station, TX: StataCorp LLC, 2017.

14. R Core Team. R: a language and environment for statistical computing. Vienna, Austria: R Foundation for Statistical Computing, https://www.R-project. org/ (2019) (accessed 22 June 2020).

15. Therneau T. A package for survival analysis in $R$ $\mathrm{R}$ package version 3.1-12, https:/CRAN. R-project.org/package $=$ survival (2020) (accessed 22 June 2020).

16. Kassambara A, Kosinski $M$ and Biecek P. survminer: drawing survival curves using 'ggplot2'. R package version 0.4 .6 , https:// CRAN.R-project.org/package $=$ survminer (2019) (accessed 22 June 2020).
17. Jin JM, Bai P, He W, et al. Gender differences in patients with COVID-19: focus on severity and mortality. Front Public Health 2020; 8: 152.

18. Harpaz R, Dahl RM and Dooling KL. Prevalence of immunosuppression among US adults, 2013. FAMA 2016; 316: 2547-2548.

19. Patel M, Chen J, Kim S, et al. Analysis of MarketScan data for immunosuppressive conditions and hospitalizations for acute respiratory illness, United States. Emerg Infect Dis 2020; 26: 1720-1730.

20. Joint Guideline British Transplant Society and the Renal Association. Guidance on the management of transplant recipients diagnosed with or suspected of having COVID19. Updated March 25th 2020, https://bts.org.uk/wp-content/ uploads/2020/03/Clinical_management_ transplant_recipients.pdf (accessed 8 May 2020).

21. Carbajo-Lozoya J, Müller MA, Kallies S, et al. Replication of human coronaviruses SARS-CoV, HCoV-NL63 and HCoV-229E is inhibited by the drug FK506. Virus Res 2012; 165: 112-117.

22. Russell B, Moss C, Rigg A, et al. COVID-19 and treatment with NSAIDs and corticosteroids: should we be limiting their use in the clinical setting? Ecancermedicalscience 2020; 14: 1023.

23. National Institute for Health and Care Excellence. COVID-19 rapid guideline: dermatological conditions treated with drugs affecting the immune response. NICE guideline [NG169], https://www.nice.org.uk/guidance/ ng169 (2020, accessed 8 May 2020).

24. American Academy of Dermatology. Guidance on the use of medications during COVID-19 outbreak, https://assets.ctfassets.net/1 ny4yoiyrqia/ PicgNuD0IpYd9MSOwab47/5e6d85324e7b5aa fed45dde0ac4ea21e/Guidance_on_medications_ AHTF_approved_April_15.pdf (2020) (accessed 22 June 2020).

25. National Institute for Health and Care Excellence. COVID-19 rapid guideline: rheumatological, autoimmune, inflammatory and metabolic disorders. NICE guideline [NG167], https://www.nice.org.uk/guidance/ng167 (2020, accessed 16 October 2020).

26. American College of Rheumatology. COVID19 clinical guidance for adult patients with rheumatic diseases. ACR COVID-19 Clinical Guidance Task Force, https://www. rheumatology.org/Portals/0/Files/ACR-COVID19-Clinical-Guidance-Summary-Patients-withRheumatic-Diseases.pdf (2020) (accessed 22 June 2020). 
27. Geleris J, Sun Y, Platt J, et al. Observational study of hydroxychloroquine in hospitalized patients with Covid-19. N Engl f Med. Epub ahead of print 7 May 2020. DOI: $10.1056 /$ NEJMoa2012410.

28. National Institute for Health and Care Excellence. COVID-19 rapid guideline: delivery of systemic anticancer treatments. NICE guideline [NG161], https://www.nice.org.uk/ guidance/ng161 (2020, accessed 24 October 2020).

29. Yang K, Sheng Y, Huang C, et al. Clinical characteristics, outcomes, and risk factors for mortality in patients with cancer and COVID-19 in Hubei, China: a multicentre, retrospective, cohort study. Lancet Oncol 2020; 21: 904-913.

30. National Institute for Health and Care Excellence. COVID-19 rapid guideline: severe asthma. NICE guideline [NG166], https://www.nice. org.uk/guidance/ng166/resources/covid19-rapidguideline-severe-asthma-pdf-66141904108741 (2020, accessed 20 May 2020).

31. Wu C, Chen X, Cai Y, et al. Risk factors associated with acute respiratory distress syndrome and death in patients with coronavirus disease 2019 pneumonia in Wuhan, China. FAMA Intern Med 2020; 180: 934-943.

32. British Society of Gastroenterology. Guidance for management of inflammatory bowel disease during the COVID-19 pandemic, https:// www.bsg.org.uk/covid-19-advice/guidance-formanagement-of-inflammatory-bowel-diseaseduring-the-covid-19-pandemic/ (2020, accessed 20 May 2020).
33. Gisondi P, Facheris P, Dapavo P, et al. The impact of the COVID-19 pandemic on patients with chronic plaque psoriasis being treated with biological therapy: the Northern Italy experience. Br f Dermatol 2020; 183: 373-374.

34. Georgakopoulos JR, Mufti A, Vender R, et al. Treatment discontinuation and rate of disease transmission in psoriasis patients receiving biologic therapy during the COVID-19 pandemic: a Canadian multicenter retrospective study. F Am Acad Dermatol 2020; 83: 1212-1214.

35. Gianfrancesco M, Hyrich KL, Al-Adely S, et al. Characteristics associated with hospitalisation for COVID-19 in people with rheumatic disease: data from the COVID-19 Global Rheumatology Alliance physician-reported registry. Ann Rheum Dis 2020; 79: 859-866.

36. Haberman R, Axelrad J, Chen A, et al. Covid-19 in immune-mediated inflammatory diseases case series from New York. N Engl F Med 2020; 383: 85-88.

37. American College of Cardiology. Chinese clinical guidance for COVID-19 pneumonia diagnosis and treatment. 7th ed. Washington, DC: American College of Cardiology, 2020.

38. Zhou F, Yu T, Du R, et al. Clinical course and risk factors for mortality of adult inpatients with COVID-19 in Wuhan, China: a retrospective cohort. Lancet 2020; 395: 1054-1062.

39. Argenziano MG, Bruce SL, Slater CL, et al. Characterization and clinical course of 1000 patients with coronavirus disease 2019 in New York: retrospective case series. BMF 2020; 369: m1996.
Visit SAGE journals online journals.sagepub.com/ home/taw

@SAGE journals 